\title{
Influence of Movement Quality on Heart Rate While Per- forming the Dance-Specific Aerobic Fitness Test (DAFT) in Preprofessional Contemporary Dancers
}

\author{
Annemiek Tiemens, MSc, 1,2,3 Rogier M. van Rijn, PhD, 1,3 Matthew A. Wyon, PhD, 2,5 \\ Emma Redding, PhD, ${ }^{4,5}$ and Janine H. Stubbe, PhD ${ }^{1,3,6}$
}

OBJECTIVES: To explore whether movement quality has influence on heart rate (HR) frequency during the dancespecific aerobic fitness test (DAFT).

METHODS: Thirteen contemporary university dance students (age $19 \pm 1.46$ yrs) underwent two trials performing the DAFT while wearing a Polar HR monitor (Kempele, Finland). During the first trial, dancers were asked to perform the movements as if they were performing on stage, whereas during the second trial, standardized verbal instructions were given to reduce the quality of movement (e.g., no need to perform technically correct pliés). The variables measured at each trial were HR for all five stages of the DAFT and HR recovery ( 1 and 2 min after finishing the DAFT), movement quality (MQ) score, and rate of perceived exertion score (RPE).

RESULTS: There were significant differences in HR between Trial 1 and Trial 2. For all stages and the resting period, HR was lower during Trial 2 ( $p<0.001)$. Also, the RPE score was significantly lower and the $M Q$ score was significantly higher, indicating a poorer performance, during Trial 2 (both $p<0.001$ ).

CONCLUSION: The results suggest that DAFT performance with lower movement quality elicits lower HR frequency and RPE during the DAFT. We recommend that specific instructions be given to participants about executing the movement sequence during the DAFT before testing commences. Also, movement quality must be

From ${ }^{1}$ Codarts Rotterdam, University of the Arts, Rotterdam. The Netherlands; 'University of Wolverhampton, Research Centre for Sport, Exercise and Performance, Wolverhampton, United Kingdom; ${ }^{3}$ Performing Artist and Athlete Research Lab (PEARL), Rotterdam, The Netherlands; ${ }^{4}$ Trinity Laban Conservatoire of Music and Dance, London, United Kingdom; ${ }^{5}$ National Institute of Dance Medicine and Science, United Kingdom; and ${ }^{6}$ Rotterdam Arts \& Sciences Lab (RASL), Rotterdam, The Netherlands.

Grant support provided by National Association of Applied Sciences SIA grant no. 2015-02-73P. The authors declare no conflicts of interest related to this study.

Address for correspondence: Annemiek Tiemens, Codarts University of the Arts, Kruisplein 26, 7th floor, 3012 CC Rotterdam. The Netherlands. Tel +31 102171045. atiemens@codarts.nl.

https://doi.org/10.21091/mppa.2018.2012

(c) 2018 Science \& Medicine. www.sciandmed.com/mppa taken into account when interpreting $\mathrm{HR}$ results from the DAFT in order to distinguish if a dancer's low HR results from good aerobic fitness or from poor performance of the movement sequence.

Med Prob/ Perform Art 2018; 33(2):77-81.

$\mathrm{D}$ ance is categorized as a high-intensity intermittent activity, since dance exercises and performances consist of short bouts of exertion followed by relatively long periods of rest. ${ }^{1-3}$ Despite the relatively high amount of recovery time, the overall intensity of exercises during classes, and variations during performance can be quite high. During center- and floorwork and performances, oxygen consumption can rise up to $60 \%$ and $80 \%$ of $\mathrm{VO}_{2 \max }$, respectively. ${ }^{1,4}$

Several studies showed that cardiorespiratory requirements, e.g., heart rate (HR) and oxygen consumption, during classes and rehearsals were not sufficient to prepare dancers for the physical demands of performances. ${ }^{5,6}$ There are often no cardiorespiratory training effects after long periods of dance training alone, since the training impulse of classes is too low due to a high amount of recovery time and a low overall heart rate. ${ }^{2,5}$ Therefore, supplemental training is desirable if a dancer needs to improve aerobic fitness in order to meet with the demands of the performance. ${ }^{2,5}$ Additionally, low levels of aerobic fitness were found to be positively related with higher numbers of injuries. ${ }^{7} \mathrm{~A}$ better aerobic fitness results in a faster recovery between two successive exercises and thus in a slower onset of fatigue, which is an indirect cause of injury. 8,9 Therefore, dancers are required to possess a certain base level of aerobic fitness in order to complete classes, rehearsals, and performances without being at risk of sustaining an injury.

Physical screening of contemporary dancers' aerobic capacity at the beginning of a new season can be very helpful to detect which dancers have low levels of aerobic fitness and might therefore be at higher risk of sustaining an injury. Originally, graded cycle ergometer tests were used to determine maximal oxygen uptake amongst dancers. ${ }^{1}$ However, recently it was found that predicting oxygen consumption in dance from an $\mathrm{HR}-\mathrm{VO}_{2}$ relationship 
derived during a cycling test was not reliable. ${ }^{10}$ Lower levels of oxygen uptake were found for similar HRs during classes when compared with the exercise test. The authors concluded that this was due to the non-steady-state characteristics of dance, which cannot be compared with data from a steady-state activity such as running or cycling. ${ }^{10} \mathrm{It}$ was found that the interval shuttle run test (ISRT) was a reliable test for intermittent sports. ${ }^{11}$ Although dance is described as an intermittent type of exercise, ${ }^{3}$ the ISRT does not lead to accurate predictions of aerobic capacity because dancers often feel restricted while running due to anatomical characteristics. ${ }^{12}$

Given that measuring aerobic fitness in dance with a graded ergometer test or ISRT is not reliable, a dance-specific aerobic fitness test (DAFT) was developed to determine aerobic fitness in dancers. ${ }^{12,13}$ The DAFT consists of a contemporary dance sequence of five stages, and at the end of each stage, HR and oxygen consumption can be measured. ${ }^{12}$ The DAFT was intended to be used as an easy-to-administer field-based assessment of aerobic fitness whereby HR measurements and oxygen uptake were used to determine fitness levels in contemporary dancers. The authors concluded that the DAFT was a valid test to determine aerobic fitness in contemporary dancers since HR frequency and oxygen uptake during stage 3 reflect those of contemporary dance classes and at the end of stage 5 mirror those of performances. ${ }^{12}$ Moreover, there was a high correlation between oxygen uptake and HR frequency $(r=0.91, p<0.001)$. The reliability of the DAFT was assessed by determining the coefficient of variation $(\mathrm{CV})$ between two separate trials. CV was between 1.4 and 6.0\% for all stages. ${ }^{12}$

Several studies have already used HR measurements during the DAFT to determine aerobic fitness in dancers. These studies found an association with injury levels and aesthetic competence. ${ }^{7,15}$ Moreover, Angioi et al. (2012) ${ }^{14}$ used the DAFT to measure the effect of supplemental training on aerobic fitness and aesthetic competence. The supplemental training resulted in lower HR frequency at the end of the DAFT. This coincided with an increase in aesthetic competence. ${ }^{14}$ However, to date no research of possible confounding factors on the outcome of the DAFT has been published. Therefore, this paper aimed to study whether differences in movement quality would influence the HR frequency during the DAFT. It was hypothesized that there is a correlation between movement quality and HR frequency. A low quality of movement during the DAFT will result in an overall lower HR than when the dancer executes the DAFT as if it was a performance.

\section{METHODS}

\section{Participants}

Thirteen contemporary dance students ( $n=13 ; 7$ males, 6 females; age $19 \pm 1.46 \mathrm{yrs}$ ) volunteered to participate in the study. The participants were either first- $(n=5,38.5 \%)$ or second-year ( $n=6,46.2 \%)$ full-time students in a bachelor's degree in dance or first-year students $(n=2,15.4 \%)$ in a bachelor's degree in dance in education at Codarts University of the Arts, Rotterdam, The Netherlands. All participants were healthy and free of injuries. Prior to testing, the participants completed a Physical Activity Readiness Questionnaire (PAR-Q) to determine whether they would be able to participate in the study, and they completed a consent form. Ethical approval was provided by the Medical Ethics Review Committee of the Academic Medical Centre Amsterdam, The Netherlands (W15_200).

\section{DAFT}

The DAFT consists of five stages of 4 minutes' duration, with a total test time of $20 \mathrm{~min}$. Every 4-min continuous stage resembles a contemporary dance sequence comprising of the same movements: lunges, pliés, jumps, and circular arm movements. Between every successive stage, there is an increase in movement tempo and movement difficulty. ${ }^{16}$ For an extensive explanation about the movement sequence of every stage, see Redding and Wyon (2010). ${ }^{16}$ The outcome measurement of the DAFT is HR frequency at the end of every stage. Furthermore, it is advised by the designers of the test to observe movement quality, although no clear guidelines are presented in the test manual. ${ }^{16}$ The following nine determinants are proposed to be used: sequencing, coordination, effort levels, travelling distance, pointed feet, consistent arm position, lunge depth (chest to thigh), jump height, and overall movement quality.

\section{Procedures}

The participants were tested on two separate trials within a period of 1 month. For the purposes of this study, a familiarization trial was not needed since all dancers were already familiar with the protocol of the DAFT and had performed the test at least once in the past year. To be sure that all dancers remembered the choreography, the dance sequence was repeated before starting the DAFT. At the end of the DAFT, participants were required to sit down for 2 minutes to rest.

The participants wore a Polar Team 2 HR monitor (Kempele, Finland) that was connected to the corresponding software on a laptop. The HR responses to the test and rest period were recorded, and markers were placed to indicate the beginning and end of the five stages and 1 and 2 minutes of rest. The markers were used to determine the mean HR during the last minute of all stages ${ }^{14}$ and after the first and second minute of rest. Next to the objective measurement of intensity (HR), a subjective measurement was used by asking the participants to grade their rate of perceived exertion (RPE) directly after stage 5 was completed by using the $6-20$ Borg-scale. ${ }^{17}$

For Trial 1, the original instructions as described in the DAFT manual ${ }^{16}$ were given to the participants: executing 
TABLE 1. HR, MQ Scores, and RPE Scores in Trial I vs Trial 2

\begin{tabular}{lcccc}
\hline & Trial I & Trial 2 & $\begin{array}{c}\text { Mean } \\
\text { Difference }\end{array}$ & $p$-Value \\
\hline HR Stage I & $122 \pm 12.85$ & $104 \pm 10.66$ & $17.38 \pm 9.62$ & $<0.001$ \\
HR Stage 2 & $135 \pm 14.04$ & $111 \pm 12.75$ & $24.23 \pm 9.8 \mid$ & $<0.001$ \\
HR Stage 3 & $165 \pm 15.34$ & $124 \pm 14.70$ & $41.00 \pm 12.52$ & $<0.001$ \\
HR Stage 4 & $181 \pm 10.07$ & $136 \pm 14.61$ & $44.46 \pm 12.96$ & $<0.001$ \\
HR Stage 5 & $190 \pm 7.13$ & $\mid 44 \pm 14.26$ & $45.08 \pm 13.28$ & $<0.001$ \\
HR Rest I & $140 \pm 7.23$ & $104 \pm 15.62$ & $36.46 \pm 13.14$ & $<0.001$ \\
HR Rest 2 & $115 \pm 8.10$ & $91 \pm 20.24$ & $23.38 \pm 17.18$ & $<0.001$ \\
MQ & $3.77 \pm 2.89$ & $19.62 \pm 3.43$ & $-15.85 \pm 4.72$ & $<0.001$ \\
RPE & $14.15 \pm 1.63$ & $9.54 \pm 1.66$ & $4.62 \pm 1.45$ & $<0.001$ \\
\hline
\end{tabular}

Data given as mean $\pm \mathrm{SD}$. HR, bpm; MQ scores range 0-45; and RPE range 6-20.

each stage as fully and accurate as possible, starting each stage at relatively the same place in space, and executing the test as if you are performing on stage. For Trial 2, the participants were instructed verbally to perform with a lower quality of movement. A standardized protocol, based on the nine determinants of movement quality, was used to make sure all participants received the same instructions. The instructions were focused on reducing movement amplitude (e.g., smaller pliés, lower jumps, and smaller travelling distance). Besides, participants were instructed to be less technically perfect when performing movements such as pliés and sautés.

To determine the quality of movement, the participants were filmed while performing the test on both occasions. The videos were used to assess the movement quality by using the nine movement observations that have been advised in the manual. ${ }^{16} \mathrm{~A}$ scientist that was blinded for the experiment scored the movement quality. Every time a participant could not maintain the same technical quality or amplitude of the movement for one of the nine variables throughout a stage, a tally mark was given. Adding the tally marks for each participant resulted in a total movement quality (MQ) score for both trials ranging from 0 (excellent movement quality) to 45 (poor movement quality).

\section{Statistical Analysis}

Data were analyzed using IBM SPSS Statistics software (ver 24, IBM-SPSS, Armonk, NY, USA). To determine if the data were normally distributed, skewness and kurtosis scores were calculated and the Kolmogorov-Smirnov normality test was applied. Paired $t$-tests were performed to determine statistical differences for $\mathrm{HR}, \mathrm{MQ}$, and RPE between the different trials. In order to explore correlations between $\mathrm{HR}, \mathrm{MQ}$, and RPE, individual differences (Trial 1 vs Trial 2) were determined for HR at the end of stage $5, M Q$, and $R P E$, resulting in a $H R_{\text {diff }}, M Q_{\text {diff, }}$ and $\mathrm{RPE}_{\text {diff }}$ score for each participant. Pearson correlation coefficient was used to explore linear correlations between $\mathrm{HR}_{\text {diff, }}, \mathrm{MQ}_{\text {diff, }}$ and $R P E_{\text {diff. }}$. Statistical significance was set at $p<0.05$ for all analyses.
TABLE 2. Different Determinants of the MQ Score for All Stages and Participants, Trial I vs Trial 2

\begin{tabular}{lccc}
\hline & Trial I & Trial 2 & p-Value \\
\hline Sequencing & $1.00 \pm 0.707$ & $3.40 \pm 0.894$ & 0.009 \\
Coordination & 0 & 0 & - \\
Effort levels & 0 & $10.80 \pm 2.683$ & 0.001 \\
Travelling distance & 0 & 0 & - \\
Pointed feet & $1.80 \pm 2.049$ & $7.40 \pm 6.804$ & 0.075 \\
Consistent arm position & $1.80 \pm 1.643$ & $7.80 \pm 5.357$ & 0.028 \\
Lunge depth (chest to thigh) & $4.00 \pm 2.550$ & $8.20 \pm 3.834$ & 0.008 \\
Jump height & $1.20 \pm 1.789$ & $7.40 \pm 6.804$ & 0.072 \\
Overall movement quality & 0 & $5.60 \pm 3.130$ & 0.016 \\
\hline
\end{tabular}

Data given as mean \pm SD .

\section{RESULTS}

\section{Heart Rate Frequency}

All participants were able to perform the movements of the DAFT and were able to complete the full test at both trials. Table 1 shows the mean $( \pm$ SD) HR per stage as well as RPE and MQ scores for Trial 1 and Trial 2.

For the HR frequency during the DAFT, a significant mean difference was found between Trial 1 and Trial 2 in all stages and the 2 minutes of recovery. HR mean differences (MD) between Trial 1 and Trial 2 ranged from 17.38 to 45.08 bpm (Table 1). HR was significantly higher during Trial 1 than during Trial 2 for all stages and the recovery period.

\section{Movement Quality and RPE}

Table 1 shows a significant reduction in movement quality between both trials as seen by a higher MQ score for Trial 2 (MD $15.85 \pm 4.72$ ) and a significant lower RPE score in Trial 2 compared to Trial 1 (MD $4.62 \pm 1.45$ ).

An overview of the determinants scored to generate the MQ scores is given in Table 2, which shows the number of observations for all stages and all participants. From Table 2 , it can be concluded that the largest difference in movement quality between Trial 1 and Trial 2 was found for the determinant effort level. A significant difference was found for sequencing, effort level, consistent arm position, lunge depth (chest to thigh), and overall movement quality. For the determinants pointed feet and jump height, the results were non-significant. Strikingly, the determinant lunge depth (chest to thigh) was scored often during both trials, whereas coordination and travelling distance were not used in either trial.

No significant correlations were found between $\mathrm{HR}_{\text {diff }}$ and $\mathrm{MQ}_{\text {diff }}(r=0.394, p=0.183)$, nor for the difference in $\mathrm{HR}_{\text {diff }}$ and $\mathrm{RPE}_{\text {diff }}(r=0.180, p=0.557)$.

\section{DISCUSSION}

The aim of this study was to assess whether movement quality is associated with the HR response during the DAFT. For Trial 1, the mean HR at the end of stage 5 was 
$190 \mathrm{bpm}$, which is $94.5 \%$ of the mean expected $\mathrm{HR}_{\text {max }}$ (220 - mean age). For Trial 2, participants reached only $71.6 \%$ of their expected $\mathrm{HR}_{\max }$. Additionally, RPE was significantly lower. This shows that both objective and subjective variables of intensity were lower in Trial 2. Next to a decreased HR and RPE, movement quality also was worse in the second trial. The results of the present study suggest that a lower quality of movement coincides with a lower HR during all stages of the DAFT and a lower subjective perceived exertion. However, due to the absence of correlations between $H R_{\text {diff }}, \mathrm{MQ}_{\text {diff }}$, and $\mathrm{RPE}_{\text {diff }}$, it remains unclear if the participant with the largest difference in HR between Trial 1 and Trial 2 was also the participant with the greatest difference in movement execution.

In order to monitor physical fitness throughout the year, comparisons between the HR results of the DAFT at different time points are required. The results of the present study show that the movements need to be performed as standardized as possible to reduce the effect of movement quality on HR frequency. Therefore, the use of clear instructions is necessary. It is suggested that multiple movement quality determinants should be observed during the DAFT. $^{12}$ These variables were sequencing, coordination, effort levels, travelling distance, pointed feet, consistent arm position, lunge depth (chest to thigh), jump height, and overall movement quality. Unfortunately, the authors do not provide further directions for the observations of these movements. As a result, it is up to the observer to determine whether a dancer performs the movements in a standardized way each time they perform the test.

Therefore, studies are needed to determine which MQ determinants are the main contributors to the HR differences found in the present study and if determinants are missing in the current observation guidelines. For example, the observer noted that one participant had a lot of movement in the trunk when doing the pliés during the test but could not attribute this to one of the determinants. The main determinants that were used most in both sessions were sequencing, pointed feet, consistent arm position, lunge depth, and jump height. Also, the largest difference in total number of observation between Trial 1 and Trial 2 was found for the determinants effort level and overall movement quality. Therefore, it is likely that poor performance on these determinants will lead to the reduction of HR frequency during the DAFT. However, no correlation between movement quality and HR frequency was found in the present study. The absence of such a correlation can be explained by the fact that every determinant could be used only once during a stage. If a participant made one mistake in the sequencing of the choreography, this was scored as one tally mark for sequencing; however, if another dancer performed a lunge that was not correct throughout the whole stage, this was also scored as one tally mark for lunge depth (chest to thigh). Both contributed equally to the MQ score, although it is to be expected that one mistake in sequencing does not contribute to a lower or higher HR, but a con- stant poor lunge would. Therefore, a clear protocol describing how to measure movement quality and how to take into account the MQ score when interpreting the HR outcome of the DAFT is necessary.

The present findings further suggest that the RPE measurements cannot be used separately from HR measurements to determine the intensity of the test. The RPE score was originally correlated to $\mathrm{HR}$, where a score of 6 equals a $\mathrm{HR}$ of $60 \mathrm{bpm}$ and a score of 20 equals a HR of $200 \mathrm{bpm} .{ }^{17}$ However, this one-to-one correlation was not found during the present study, since the $\mathrm{HR}_{\text {diff }}$ between Trial 1 and Trial 2 was not correlated to the RPE $E_{\text {diff }}$. Therefore, it is advised to use the RPE next to the HR measurements to gain insight in the dancer's perceived exertion of the test, but not to use as a single measurement of intensity.

This study has several strengths. First, it is the first paper that addresses the influence of movement quality on HR during the DAFT. Second, by using the Polar Team 2 system, HR frequency was continually recorded throughout the test. Thereby, the mean HR over the last minute of every stage could be determined, resulting in a reliable and objective value. Another strength of the study was that it included a homogeneous group of young, high-level contemporary dancers. However, the study also had some limitations. First, only a small number of participants was included, which might contribute to the lack of correlations between HR, MQ, and RPE scores. Second, the two trials were separated by a period of 1 month maximum. It is questionable if training effects occurred within this period. However, several studies suggested that performing dance training alone is not sufficient to improve aerobic fitness. ${ }^{5,6}$ Moreover, a study by Angioi et al. ${ }^{14}$ showed a decrease in HR of $19 \mathrm{bpm}$ after a 6-week supplemental training intervention, whereas the control groups only improved by $11 \mathrm{bpm}$. In the present study, the difference in $\mathrm{HR}$ at the end of the DAFT was $45 \mathrm{bpm}$. The students in the current study received their regular educational program over the 1-month period. Therefore, the difference in HR cannot be explained by an improvement in aerobic fitness alone. Another limitation of this study was the lack of randomization of the participants and the two trials. Ideally, this study needs to be repeated and participants should be assigned to two groups randomly, with one group performing Trial 1 first and Trial 2 second, and a second group performing Trial 2 first and Trial 1 second. Finally, due to the exploratory nature of the study, no adjustment for the multiple comparison issues was made.

\section{Conclusions}

Our study showed that movement quality influences HR during the DAFT. Therefore, it is important to give clear instructions about the movement quality to the participants before the test starts. Furthermore, movement quality should be scored during the test, and standardized observation guidelines are required as well as clear instructions on how to use the MQ score while interpreting the HR out- 
come of the DAFT. When using HR as the only outcome measurement of the DAFT, it might be concluded that the dancer has a good aerobic fitness, but this might also be the result of poor performance of the movement sequence.

\section{REFERENCES}

1. Schantz PG, Åstrand P. Physiological characteristics of classical ballet. Med Sci Sports Exerc. 1984;16(5):472-6. https:/ / doi.org/ 10.1249/00005768-198410000-00009

2. Rodrigues-Krause J, Krause M, Reischak-Oliveira Á. Cardiorespiratory considerations in dance: from classes to performances. J Dance Med Sci. 2015;19(3):91-102. https:/ / doi.org/10.12678/ 1089-313X.19.3.91

3. Wyon M. Cardiorespiratory training for dancers. J Dance Med Sci. 2005;9(1):7-12.

4. Rodrigues-Krause J, dos Santos Cunha G, Alberton CL, et al. Oxygen consumption and heart rate responses to isolated ballet exercise sets. J Dance Med Sci. 2014;18(3):99-105. https:// doi.org/10.12678/1089-313X.18.3.99

5. Wyon MA, Abt G, Redding E, et al. Oxygen uptake during modern dance class, rehearsal, and performance. J Strength Cond Res. 2004;18(3):646-9. https:/ / doi.org/10.1519/13082.1

6. Wyon MA, Redding E. Physiological monitoring of cardiorespiratory adaptations during rehearsal and performance of contemporary dance. J Strength Cond Res. 2005;19(3):611-4. https:// doi.org/10.1519/14233.1

7. Twitchett E, Brodrick A, Nevill AM, et al. Does physical fitness affect injury occurrence and time loss due to injury in elite vocational ballet students? J Dance Med Sci. 2010;14(1):26-31.

8. Wyon MA, Koutdeakis Y. Muscular fatigue: considerations for dance. J Dance Med Sci. 2013;17(2):63-9. https://doi.org/ 10.12678/1089-313X.17.2.63
9. Liederbach M, Schanfein L, Kremenic IJ. What is known about the effect of fatigue on injury occurrence among dancers? $J$ Dance Med Sci. 2013;17(3):101-8. https://doi.org/10.12678/ 1089-313X.17.3.101

10. Redding E, Wyon M, Shearman J, Doggart L. Validity of using heart rate as a predictor of oxygen consumption in dance. $J$ Dance Med Sci. 2004;8(3):69-72.

11. Lemmink KAPM, Visscher C, Lambert MI, Lamberts RP. The interval shuttle run test for intermittent sport player: evaluation of reliability. J Strength Cond Res. 2004;18(4):821-7. https:/ / doi. org / 10.1519/13993.1

12. Wyon M, Redding E, Abt G, et al. Development, reliability, and validity of a multistage dance specific aerobic fitness test (DAFT). J Dance Med Sci. 2003;7(3):80-4.

13. Redding E, Weller P, Ehrenberg S, et al. The development of a high intensity dance performance fitness test. J Dance Med Sci. 2009;13(1):3-9.

14. Angioi M, Metsios G, Twitchett EA, et al. Effects of supplemental training on fitness and aesthetic competence parameters in contemporary dance: a randomised controlled trial. Med Probl Perform Art. 2012;27(1):3-8.

15. Angioi M, Metsios GS, Twitchett E, et al. Association between selected physical fitness parameters and aesthetic competence in contemporary dancers. J Dance Med Sci. 2009;13(4):115-23.

16. Redding E, Wyon M. Dance-specific fitness test. London: Trinity Laban Conservatoire of Music and Dance; 2010.

17. Borg GAV. Psychophysical bases of perceived exertion. Med Sci Sports Exerc. 1982;14(5):377-81. https://doi.org/10.1249/ 00005768-198205000-00012

Received 3-Apr-2017, accepted 11-Jan-2018

Published online 1-June-2018

https: / / doi.org/10.21091/mppa.2018.2012 